\title{
Enhanced diagnosis of HIV-1-associated tuberculosis by relating T-SPOT.TB and
} CD4 counts

\author{
T. Oni*,\#, J. Patel", H.P. Gideon*, R. Seldon*, K. Wood*, Y. Hlombe*, \\ K.A. Wilkinson ${ }^{\star,+}$, M.X. Rangaka ${ }^{\star, \varsigma, f}$, M. Mendelson ${ }^{\star *}$ and R.J. Wilkinson*,\#,+
}

ABSTRACT: The sensitivity of the tuberculin skin test is impaired in HIV-1-infected persons. Enzyme-linked immunospot-based detection of immune sensitisation may be less affected. Furthermore, the quantitative response can be related to the CD4 count, potentially improving specificity for active disease.

The T-SPOT.TB assay was performed on HIV-1-infected participants, 85 with active tuberculosis (TB) and 81 healthy patients (non-TB). The ratio of the sum of the 6-kDa early secretory antigenic target and culture filtrate protein 10 response to the CD4 count (spot-forming cell (SFC)/CD4) was calculated.

Using the manufacturer's guidelines, active TB was diagnosed with $76 \%$ sensitivity and $53 \%$ specificity. Using an SFC/CD4 ratio of 0.12 , sensitivity $(80 \%)$ and specificity $(62 \%)$ improved. The quantitative $\mathrm{T}$-cell response increased with increasing smear-positivity in the active TB group $(p=0.0008)$. In the non-TB group, the proportion of persons scored positive by T-SPOT.TB assay was lower in the group with a CD4 count of $<200$ cells $\cdot \mathrm{mm}^{-3}(p=0.029)$.

The ratio of the summed T-cell response to CD4 count improved the diagnostic accuracy of the T-SPOT.TB assay in HIV-1-infected persons, and a ratio of SFC/CD4 of $>0.12$ should prompt investigation for active disease. A strong association between the degree of sputum positivity and T-SPOT.TB score was found. The sensitivity of the T-SPOT.TB assay in active disease may be less impaired by advanced immunosuppression.

\section{KEYWORDS: Diagnosis, HIV-1, interferon- $\gamma$, management, Mycobacterium tuberculosis}

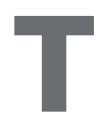
he World Health Organization's strategy for halting the spread of tuberculosis (TB) outlines amongst its targets the improvement of TB diagnosis in HIV-1 (HIV)-infected persons. Africa accounts for $>31 \%$ of the global burden of TB and had an estimated 1.39 million HIV-infected TB cases and 480,000 deaths in 2005 [1]. It is well recognised that CD4 T-cell depletion increases the risk of developing HIV-associated TB $[2,3]$, with the risk of incident TB shown to double within the first year following seroconversion [4]. The performance of widely used TB diagnostic tests, such as the tuberculin skin test (TST) and sputum microscopy, in HIV-infected patients is compromised [5-7].

Interferon- $\gamma$ release assays (IGRAs) are in vitro immunodiagnostic tests that measure the T-cell interferon- $\gamma$ response to Mycobacterium tuberculosisspecific antigens. Studies have assessed the power of IGRA-based analysis to detect latent TB infection (LTBI) in HIV-co-infected persons in endemic
TB and HIV settings [8, 9]. A head-to-head comparison of IGRAs demonstrated that the T-SPOT. $T B$ assay gave a higher proportion of positive results than the QuantiFERON ${ }_{\circledast}$ TB-Gold In-Tube (QFT-GIT) assay in HIV-infected adults [10]. A larger comparison of RANGAKA et al. [11], in a setting with high endemic TB, also suggested that the T-SPOT.TB assay was less impaired in advanced immunosuppression [11]. However, these tests, as presently interpreted, do not permit distinction between LTBI and active disease.

A pilot study by the Immunology Group Laboratory (Institute of Infectious Diseases and Molecular Medicine, Faculty of Health Sciences, University of Cape Town, Cape Town, South Africa) suggested a method of detecting active TB in HIV-infected patients by summing the enzyme-linked immunospot (ELISPOT) response to TB-specific antigens (6-kDa early secretory antigenic target (ESAT-6) and culture filtrate protein 10 (CFP-10)) and dividing this by the

\section{AFFILIATIONS}

*Institute of Infectious Diseases and Molecular Medicine, Faculty of Health Sciences, and

${ }^{\S}$ Infectious Disease Epidemiology Unit, School of Public Health, University of Cape Town, and **Division of Infectious Diseases and HIV Medicine, Dept of Medicine Groote Schuur Hospital, Cape Town, South Africa.

\#Division of Medicine, Imperial College London,

${ }^{+}$National Institute for Medical

Research, and

${ }^{f}$ London School of Hygiene and

Tropical Medicine, London, and

"Brighton and Sussex University

Hospital NHS Trust, Brighton, UK.

CORRESPONDENCE

T. Oni

3.03 Wolfson Pavillion,

Institute of Infectious Diseases and

Molecular Medicine,

Faculty of Health Sciences

University of Cape Town

Observatory 7925

Cape Town

South Africa

E-mail: tolullahoni@doctors.org.uk

Received:

Oct 292009

Accepted after revision:

Jan 062010

First published online:

Jan 142010 
CD4 cell count [12]. A ratio of $>1$ strongly suggested active disease. As these preliminary findings employed an in-house IGRA, a larger study was designed, using the ratio of the summed ELISPOT count from the T-SPOT.TB assay divided by the CD4 count for diagnosing active TB, and including a robust group of non-TB HIV-infected patients as controls.

\section{METHODS}

\section{Study location and design}

The study site at Ubuntu TB/HIV clinic is located in Khayelitsha, a peri-urban township near Cape Town (South Africa) with a population of $>400,000$. Khayelitsha has an exceptionally high burden of HIV and TB (1,612 per 100,000 population in 2005) [12], with $\sim 67 \%$ of TB being HIV-related. A cross-sectional study design was employed, sampling HIVinfected patients with active TB and HIV-infected persons without evidence of active TB as controls.

\section{Participants}

Written informed consent was obtained from all participants, and the study was approved by the University of Cape Town Research Ethics Committee (REC 012/2007; Cape Town, South Africa). All 166 participants were antiretroviral therapy (ART)naive at the time of recruitment. A total of $85 \mathrm{HIV}$-infected TB patients with culture-positive TB disease were recruited from the clinic prior to starting anti-TB chemotherapy. These patients had presented to the clinic with signs and symptoms of TB. A total of $81 \mathrm{HIV}$-infected healthy participants were enrolled from the pre-ART HIV clinic, with no symptoms of active TB using a symptom screen (any cough, night sweats, loss of weight and loss of appetite). All healthy participants (non-TB group) were induced sputum-, smear- and TB culturenegative and showed no radiological features of TB. Persons enrolled into this group underwent a TST using 2 tuberculin units of Siebert purified protein derivative of tuberculin RT23 injected intradermally into the volar aspect of the forearm. All persons with a skin induration diameter of $\geqslant 5 \mathrm{~mm}$ were offered and started on isoniazid preventive therapy (IPT) after whole blood was collected for IGRA. No participant had previously received IPT. A history of previous TB within 3 months of recruitment was an exclusion criterion.

At the point of recruitment, questionnaires were completed and blood samples collected for CD4 counts and T-SPOT.TB assays. Persons with a CD4 count of $<200$ cells $\cdot \mathrm{mm}^{-3}$ were referred to the ART clinic to start treatment as per national guidelines. The ratio of the summed ESAT- 6 and CFP-10 response to CD4 count was calculated, and receiver operating characteristic (ROC) curve analysis was conducted on results.

\section{Peripheral blood mononuclear cell preparation}

Peripheral blood mononuclear cells (PBMCs) were extracted from heparinised whole blood within $4 \mathrm{~h}$ of collection. PBMCs were separated from the whole blood using the Ficoll-Paque ${ }^{\mathrm{TM}}$ (GE Healthcare, Chalfont St Giles, UK) gradient technique and stored in liquid nitrogen for batched T-SPOT.TB analysis.

\section{ELISPOT assay}

Laboratory workers were blinded to the clinical status of participants. The ELISPOT assay was performed using the T-SPOT.TB kit according to the manufacturer's instructions
[13]. Viable PBMCs $\left(2.5 \times 10^{5}{\text { cells } \cdot w^{-1}}^{-1}\right)$ in serum-free medium were added to the pre-coated plates and stimulated with the provided antigens and controls for $16-20 \mathrm{~h}$ at $37^{\circ} \mathrm{C}$ under $5 \%$ carbon dioxide. After incubation, the plates were developed and the number of spots counted using an ImmunoSpot 3.2 reader (Cellular Technology, Cleveland, $\mathrm{OH}, \mathrm{USA}$ ) and verified manually. A positive response was defined, irrespective of the phytohaemagglutinin (PHA) positive control response, as $>6$ spot-forming cells (SFCs) well $^{-1}$ more than the negative control (if the negative control contained 0-5 SFCs.well ${ }^{-1}$ ) in either ESAT-6 or CFP-10 wells, or at least double the negative control spot count where the negative control had 6-10 SFCs.well ${ }^{-1}$. A negative response was recorded if the above criteria were not met and the positive control was valid. An indeterminate response was recorded in the presence of $<20 \mathrm{SFCs} \cdot \mathrm{well}^{-1}$ in the PHA well when ESAT-6/CFP-10 wells were nonreactive $\left(<6\right.$ SFCs $\cdot$ well $\left.^{-1}\right)$ or in the presence of a negative control count of $>10$ SFCs $\cdot$ well ${ }^{-1}$.

\section{TB microscopy and culture}

The sputum collection, microscopy and culture were performed at a national reference laboratory as part of the national TB programme. The non-TB participants underwent studyspecific sputum collection and processing.

\section{Statistical methods}

With a sample size of 166 , the aim was to detect a $30 \%$ increase in the proportion of positive T.SPOT-TB result in the active versus non-TB group with $95 \%$ power and a 5\% level of significance. Predictors of disease status were analysed by logistic regression using culture positivity or negativity as the response variable. The model was built both manually and using the stepwise method, and variables considered to be associated with active $\mathrm{TB}$, including age, sex and previous $\mathrm{TB}$ history, were included. Confounding variables and outlying and influential observations were identified and possible effect-modification assessed. ROC analysis was conducted to determine sensitivity and specificity at varying cut-off values of the T-SPOT.TB assay. The ROC curves of the T-SPOT.TB assay and the ratio of SFCs to CD4 count were compared by assessing equivalence of areas under the curve. Post hoc analysis was performed by comparing sputum smear microscopy results to the quantitative T-cell response using scatter plots and the Kruskal-Wallis test to compare medians. All p-values were two-sided with an $\alpha$ of 0.05 . All data were analysed using STATA 10.0 (StataCorp, College Station, TX, USA).

\section{RESULTS}

\section{Baseline characteristics of study participants}

Table 1 summarises the baseline characteristics of participants. Of the 166 participants, 63\% were female. TB patients were significantly more likely to be older and male with a previous history of TB. All TB patients were TB culture-positive, with 34 $(40 \%)$ being smear-negative (median time to culture positivity 17 days). Stratification by CD4 count showed that $44 \%$ of TB patients with a CD4 count of $<200$ cells $\cdot \mathrm{mm}^{-3}$ were smearnegative compared to $32 \%$ of patients with a CD4 count of $>200$ cells $\cdot \mathrm{mm}^{-3}$, although this was nonsignificant $(\mathrm{p}=0.3)$. 


\begin{tabular}{|c|c|c|c|c|}
\hline \multicolumn{5}{|l|}{ Sex } \\
\hline Female & $105(63)$ & $59(73)$ & $46(54)$ & 0.012 \\
\hline Male & $61(37)$ & $22(27)$ & $39(46)$ & \\
\hline \multicolumn{5}{|l|}{ Previous TB } \\
\hline Yes & $38(23)$ & $8(10)$ & $30(35)$ & \\
\hline Missing data & $5(3)$ & $1(1)$ & $4(5)$ & \\
\hline \multicolumn{5}{|l|}{ BCG scar } \\
\hline No & $111(67)$ & $56(69)$ & $55(65)$ & 0.272 \\
\hline Yes & $49(29)$ & $24(30)$ & $25(29)$ & \\
\hline Missing data & $6(4)$ & $1(1)$ & $5(6)$ & \\
\hline \multicolumn{5}{|l|}{ CD4 count cells $\cdot \mathrm{mm}^{-3}$} \\
\hline$<100$ & $31(19)$ & $6(8)$ & $25(30)$ & $<0.0001$ \\
\hline 100-199 & $46(28)$ & $14(17)$ & $32(37)$ & \\
\hline 200-299 & $34(20)$ & $23(28)$ & $11(13)$ & \\
\hline$\geqslant 300$ & $53(32)$ & $36(44)$ & $17(20)$ & \\
\hline Missing data & $2(1)$ & 2 (3) & $0(0)$ & \\
\hline Age yrs & 33 (28-39) & $30(26-36)$ & $35(29-41)$ & 0.0002 \\
\hline CD4 count cells $\cdot \mathrm{mm}^{-3}$ & $221.5(116.6-364.5)$ & $291(189-436)$ & 155 (73-259) & $<0.0001$ \\
\hline BMI $\mathbf{k g} \cdot \mathbf{m}^{-2}$ & $22.7(19.8-26.2)$ & $24.2(21.6-29.0)$ & $21.0(19.0-23.0)$ & $<0.0001$ \\
\hline
\end{tabular}

Data are presented as $n$ (\%) or median (interquartile range), unless otherwise indicated. TB: tuberculosis; BCG: bacille Calmette-Guérin; BMI: body mass index. Bold indicates significant $p$-values.

\section{T-SPOT.TB results}

Among the 166 participants, the T-SPOT.TB assay was positive in $92(55.4 \%)$ overall. The median number of SFCs was higher in the active ( $56 \mathrm{SFCs} \cdot 10^{6} \mathrm{PBMCs}^{-1}$; interquartile range (IQR) 24-184 SFCs $\cdot 10^{6} \mathrm{PBMCs}^{-1}$ ) versus non-TB group (20 SFCs $\cdot 10^{6}$ $\mathrm{PBMCs}^{-1}$; IQR $4-60$ SFCs $\left.\cdot 10^{6} \mathrm{PBMCs}^{-1}\right)(\mathrm{p}<0.0001)$. There was a significantly higher proportion of T-SPOT.TB-positive results in the active TB versus non-TB group ( 68 versus $42 \%$, respectively; $\mathrm{p}=0.001$ ) as shown in figure $1 \mathrm{a}$ and $\mathrm{b}$. There was a $5 \%$ indeterminate result rate (median CD4 count $154.5 \mathrm{cells} \cdot \mathrm{mm}^{-3}$, IQR 80.5-209.5 cells $\cdot \mathrm{mm}^{-3} ; \mathrm{n}=8$ ), all due to negative control spot counts of $>10$ SFCs, with a nonsignificant trend towards an increased rate in those with CD4 counts of $<200$ cells $\cdot \mathrm{mm}^{-3}$ (6.5 versus $3.3 \%$; $\mathrm{p}=0.349$ ). Of the smear-negative culture-positive $\mathrm{TB}$ patients, $71 \%$ were T-SPOT.TB-positive.

\section{SFC/CD4 ratio}

Using the T-SPOT.TB assay, the SFC/CD4 ratio was calculated by dividing the total number of SFCs in excess of that of the negative control by the CD4 count. Overall values ranged $-0.48-$ 78. The median ratio in the active TB group (0.42; IQR 0.15-1.67) was significantly higher $(\mathrm{p}<0.001)$ than in the non-TB group (median 0.079; IQR 0.02-0.21), as shown in figure 1c and d.

\section{ROC analysis}

ROC curve analysis was conducted on the results, including those with indeterminate values, using the TB and non-TB arms as comparator groups. Using the manufacturer's cut-off for the
T-SPOT.TB assay, the sensitivity for diagnosis of active TB was $76 \%$ (95\% CI 70-83\%), with a specificity of 53\% (95\% CI 46-61\%) and positive likelihood ratio of 1.63. Using a cut-off of 0.12 for the SFC/CD4 ratio, the sensitivity was $80 \%$ (95\% CI 74-86\%) and the specificity $62 \%$ (95\% CI 56-73\%), with a likelihood ratio of 2.1. Lowering the T-SPOT.TB cut-off to achieve a similar sensitivity resulted in a lower specificity (tables 2 and 3). The likelihood ratios of both the SFC/CD4 ratio and T-SPOT.TB assay were low at the chosen cut-off values, although, at any given sensitivity or specificity, the SFC/CD4 ratio showed superior likelihood ratios. The positive predictive value (PPV) was greater using the SFC/CD4 ratio than the T-SPOT.TB assay (70 versus $63 \%$ ). Importantly, the negative predictive value (NPV) for the SFC/CD4 ratio was also higher than that for the T-SPOT.TB assay (75 versus 67\%). The area under the curve was significantly greater using the SFC/CD4 ratio than the T-SPOT.TB assay $(p=0.001)$, as shown in figure $1 e$.

\section{Risk factors for active TB and T-SPOT.TB positivity}

On univariate analysis, there was a positive association between active TB and older age, lower BMI, male sex, lower CD4 count, history of previous TB, a positive T-SPOT.TB result and an SFC/CD4 ratio of $>0.12$.

On multivariate analysis, a CD4 count of $>200$ cells $\cdot \mathrm{mm}^{-3}$ (odds ratio (OR) 0.24; $\mathrm{p}=0.001)$, body mass index (OR 0.85; $\mathrm{p}=0.001)$, older age (OR 1.04; $\mathrm{p}=0.021)$ and the $\mathrm{SFC} / \mathrm{CD} 4$ ratio (OR 5.99; $\mathrm{p}<0.0001$ ) were associated with active TB (table 4). Significant 

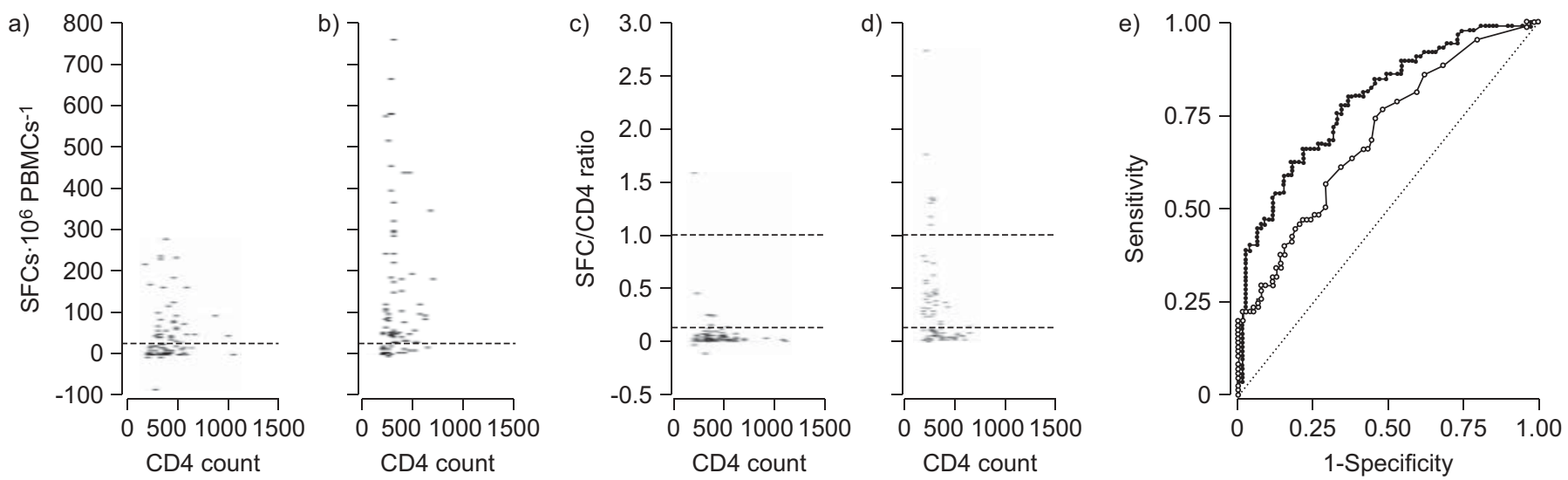

FIGURE 1. Scatter plots showing a, b) T-SPOT.TB quantitative data, and c, d) spot-forming cell (SFC)/CD4 ratio in: $a, c)$ the non-tuberculosis (non-TB) group; and b, d) the active TB group. Values above the cut-offs were regarded as positive for the T-SPOT.TB assay and SFC/CD4 ratio. At the manufacturer's cut-off of 24 SFCs-10 6 peripheral blood mononuclear cells (PBMCs) ${ }^{-1}$ (-------), the T-SPOT.TB assay was noted to give a high proportion of positive results compared to the SFC/CD4 ratio in the non-TB group. The SFC/CD4 results highlight an increase in sensitivity in distinguishing active disease from latent infection when the cut-off (-----) is lowered from 1 to 0.12 . e) Receiver operating characteristic $(\mathrm{ROC})$ curves for the T-SPOT.TB assay $(\bigcirc)$ and SFC/CD4 ratio $(\bullet)(\cdots \cdots \cdots . .$. . reference). The ratio of the area under the ROC curve was 0.790 for the SFC/CD4 ratio and 0.697 for the T-SPOT.TB assay.

predictors of a positive T-SPOT.TB result were older age (relative risk (RR) 2.2; $\mathrm{p}=0.04$ ), history of previous TB (RR 2.8; $\mathrm{p}=0.033)$ and culture-positive TB (RR 3.2; $\mathrm{p}=0.024)$. There was no significant confounding or interaction between variables.

\section{T-cell response and degree of immunosuppression}

Overall, there was no significant correlation between the IFN- $\gamma$ SFC response and the degree of immunosuppression, as measured by the CD4 count $\left(r_{S}=-0.022 ; p=0.78\right)$. However, when results were stratified by $\mathrm{CD} 4$ category and TB disease status, a CD4 count of $>200$ cells $\cdot \mathrm{mm}^{-3}$ was found to be associated with a positive T-SPOT.TB result in the non-TB group $(\mathrm{p}=0.029)$, implying that advanced immunosuppression impairs the performance of the T-SPOT.TB test in this group. Figure 2 shows an increasing proportion of T-SPOT.TB positive results with increasing CD4 count. Interestingly, this was not the case in the active TB disease group $(p=0.939)$, implying that the sensitivity of the TSPOT.TB test is impaired by advanced immunosuppression in the non-TB group but less affected in active TB.

\section{Secondary analysis}

Bacillary load and T-cell response

A post hoc analysis was conducted, relating the degree of sputum positivity in active TB to the quantitative T-SPOT.TB assay response. A significant association was found with the bacillary load, as estimated by the degree of sputum smear microscopy positivity (fig. 3). There was a positive bacillary load-immune response relationship, with a significant increase in quantitative $\mathrm{T}$-cell response with increasing smear positivity $(p=0.0008 ;$ Kruskal-Wallis test). In order to further explore this relationship, the correlation between the immune response and the number of days to culture positivity was examined as an alternative surrogate marker of bacillary load. This showed a nonsignificant negative correlation between SFCs and days to culture positivity $\left(r_{S}=-0.12 ; p=0.29\right)$, although, notably, $90 \%$ of specimens that became culture positive after a median of 11 days showed counts of $<290$ SFCs $\cdot 10^{6} \mathrm{PBMCs}^{-1}$ (the 90th percentile of SFCs), as shown in figure 4.

\section{DISCUSSION}

A novel ratio technique of diagnosing active TB was assessed in an HIV-co-infected population in a setting with high endemic TB by relating the total number of SFCs from the T-SPOT.TB assay to CD4 count. Other diagnostic studies assessing the diagnosis of active TB have been conducted using the QFT-GIT, noncommercial in-house assays and noncommercial ELISPOT assays in predominantly HIV-negative populations, or in settings with low TB endemicity $[12,14-20]$. The T-SPOT.TB

TABLE 2 Receiver operating characteristic curve analysis at varying cut-off values for the T-SPOT.TB assay

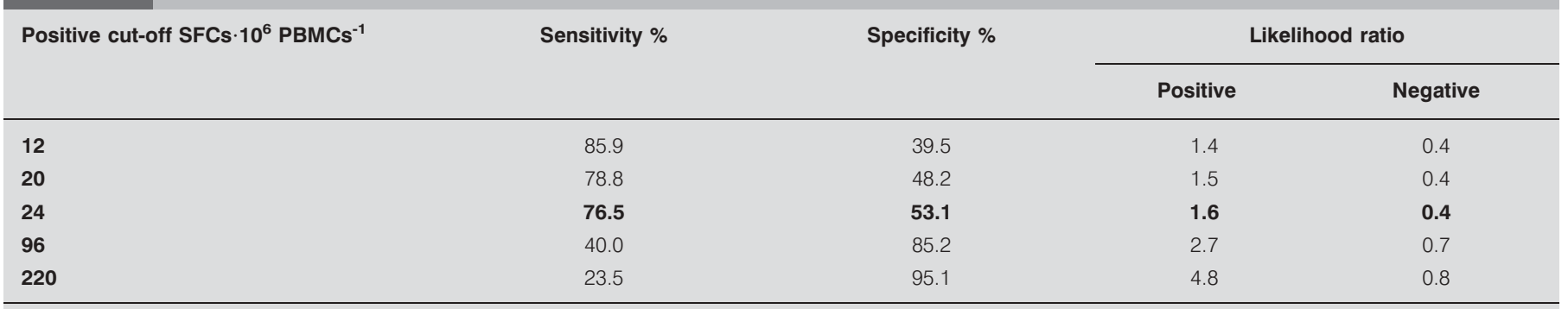

SFC: spot-forming cell; PBMC: peripheral blood mononuclear cell. Bold indicates the manufacturer's T-SPOT.TB cut-off value. 


\begin{tabular}{|c|c|c|c|c|}
\hline \multirow[t]{2}{*}{ Positive cut-off SFC/CD4 ratio } & \multirow[t]{2}{*}{ Sensitivity \% } & \multirow[t]{2}{*}{ Specificity \% } & \multicolumn{2}{|c|}{ Likelihood ratio } \\
\hline & & & Positive & Negative \\
\hline 0.04 & 91.8 & 38.0 & 1.5 & 0.2 \\
\hline 0.08 & 85.9 & 50.6 & 1.7 & 0.3 \\
\hline 1.00 & 38.8 & 97.5 & 15.3 & 0.6 \\
\hline 1.793 & 22.4 & 98.7 & 17.7 & 0.8 \\
\hline
\end{tabular}

SFC: spot-forming cell. Bold indicates the chosen cut-off ratio of 0.12 .

assay was used since previous studies have reported the T-SPOT.TB assay to show better sensitivity than the QFT-GIT test in HIV-infected persons in high-prevalence settings such as the present one $[10,11]$.

The T-SPOT.TB assay diagnosed active TB disease with a sensitivity of $76 \%$ and a specificity of $53 \%$. The SFC/CD4 ratio diagnosed $\mathrm{TB}$ disease with greater sensitivity at a ratio of $>0.12$, indicating a six-fold higher risk of $\mathrm{TB}$ disease on multivariate analysis. The sensitivity of the SFC/CD4 ratio in diagnosing active TB remained high in advanced immunosuppression. Therefore, in practice, in an HIV-infected patient with an SFC/CD4 ratio of $>0.12$, active TB should be strongly suspected and investigated, whereas a positive T-SPOT.TB result should prompt consideration of LTBI treatment. In those with an SFC/CD4 ratio of $<0.12$, although active TB is not excluded, they should be considered for treatment of LTBI. However, we acknowledge that an NPV of $75 \%$ means that $25 \%$ of active TB cases would test negative using this method (33\% using the T-SPOT.TB assay), potentially limiting its use in clinical practice.

In the smear-negative culture-positive patients, the median time to culture positivity was 17 days. Importantly, the time to diagnosis could potentially have been shortened by 2 weeks using the T-SPOT.TB assay, with the public health implications including reducing infectivity and transmission rates, as well as potentially decreasing morbidity and mortality.

In the non-TB group, a CD4 count of $<200$ cells $\cdot \mathrm{mm}^{-3}$ was associated with impaired test performance. A possible reason for this is that the higher bacillary load associated with having TB disease drives a positive TB-specific antigen response regardless of CD4 count, resulting in relatively less impaired test performance. By contrast, the positive responses in the non-TB group would be less intense due to lower bacillary load, making test accuracy susceptible to impairment in advanced immunosuppression. This hypothesis is supported by the present data, by both the presence of a strong bacillary loadimmune response association and the raw data showing higher responses in the active TB group. To our knowledge, this is one of the clearest bacillary load-immune response relationships to be demonstrated in humans, using sputum smear microscopy to estimate bacillary load. The results showed a negative correlation between the immune response and number of days to culture positivity that, although statistical significance was not found, is worthy of further investigation.

Previous studies have suggested that the infectious load is associated with the magnitude of the T-cell response. HiLl et al.

TABLE 4 Univariate and multivariate logistic regression analysis for culture-positive tuberculosis (TB) disease

\begin{tabular}{|c|c|c|c|c|}
\hline & \multicolumn{2}{|c|}{ Univariate analysis } & \multicolumn{2}{|c|}{ Multivariate analysis } \\
\hline & OR (95\% Cl) & p-value & OR $(95 \% \mathrm{Cl})$ & $\mathrm{p}$-value \\
\hline Male sex & $2.27(1.19-4.35)$ & 0.013 & $0.75(0.29-1.96)$ & 0.562 \\
\hline BMI & $0.84(0.77-0.91)$ & $<0.0001$ & $0.85(0.78-0.93)$ & 0.001 \\
\hline CD4 $>200$ cells $\cdot \mathrm{mm}^{-3}$ & $0.16(0.08-0.32)$ & $<0.0001$ & $0.24(0.11-0.54)$ & 0.001 \\
\hline \multicolumn{5}{|l|}{ Positive result } \\
\hline T.SPOT.TB & $2.72(1.52-4.86)$ & 0.001 & $0.81(0.31-2.11)$ & 0.660 \\
\hline SFC/CD4 ratio & $6.90(3.42-13.91)$ & $<0.0001$ & $5.99(2.61-13.77)$ & $<0.0001$ \\
\hline BCG scar presence & $1.35(0.77-2.36)$ & 0.291 & $1.00(0.42-2.41)$ & 1.000 \\
\hline Contact with TB & $1.30(0.81-2.11)$ & 0.282 & $1.43(0.68-2.99)$ & 0.346 \\
\hline
\end{tabular}

BMI: body mass index; SFC: spot-forming cell; BCG: bacille Calmette-Guérin. Bold indicates significant ORs and their p-values. 


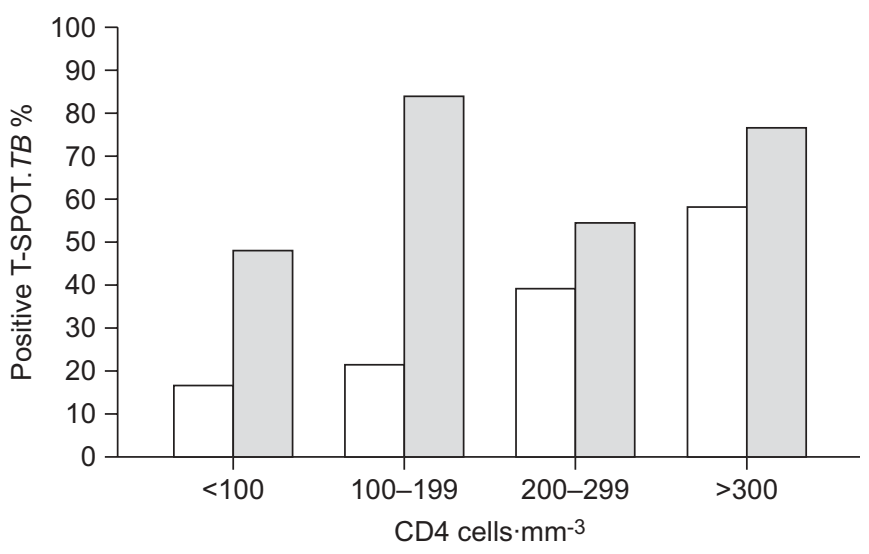

FIGURE 2. Histogram showing increasing proportion of positive T-SPOT.TB results with increasing CD4 count in the non-tuberculosis (non-TB; $\square$ ) but not the active TB group ( $(\square)$.

[21] showed that the quantitative ELISPOT response reflected the probable infecting dose of $M$. tuberculosis, measured by gradient of exposure to smear- and culture-positive TB patients. RiBEIRO et al. [22] also hypothesised that IFN- $\gamma$ producing T-cells might be related to bacterial load, determined using surrogate disease severity markers of low BMI and presence of cavitatory disease. In these two studies, participants were predominantly HIV-uninfected. These, and the present, data suggest that the T-cell response could be used as a surrogate marker for treatment response, disease relapse or monitoring progression from latent to active disease. A small pilot study conducted in Uganda by GoleTTI et al. [23] showed potential utility by demonstrating that region of difference $1 \mathrm{~T}$-cell responses decrease in HIV/TB patients after successful therapy for TB. AIKEN et al. [24] achieved a similar conclusion in a predominantly HIV-uninfected cohort. The present results suggest that the same might be true for HIV-infected persons. However RIBEIRO et al. [22] found high inter-patient variability in quantitative results, potentially limiting the use of post-treatment immune response assessment in practice.

The SFC/CD4 cut-off value used in the present study is lower than that found in a similar pilot study conducted by the Immunology Group Laboratory [12]. In order to further investigate this, a parallel comparison of the in-house assay to the T-SPOT.TB assay was conducted, and it was found that the in-house assay scored a greater proportion positive with a higher number of SFCs (data not shown). This factor contributes to the difference in ratios.

The present study has several strengths. The definition of the control non-TB group, who were both asymptomatic and gave two sputum culture-negative TB results, is very robust. In this HIV-infected population known to have a high prevalence of asymptomatic subclinical TB, we felt that the definition of nonTB persons had to be actively sought through comprehensive screening. In addition, this is the largest study to assess the ability of the T-SPOT.TB assay and the SFC/CD4 ratio to differentiate active from latent TB in an HIV-infected population in a setting with high endemic TB.

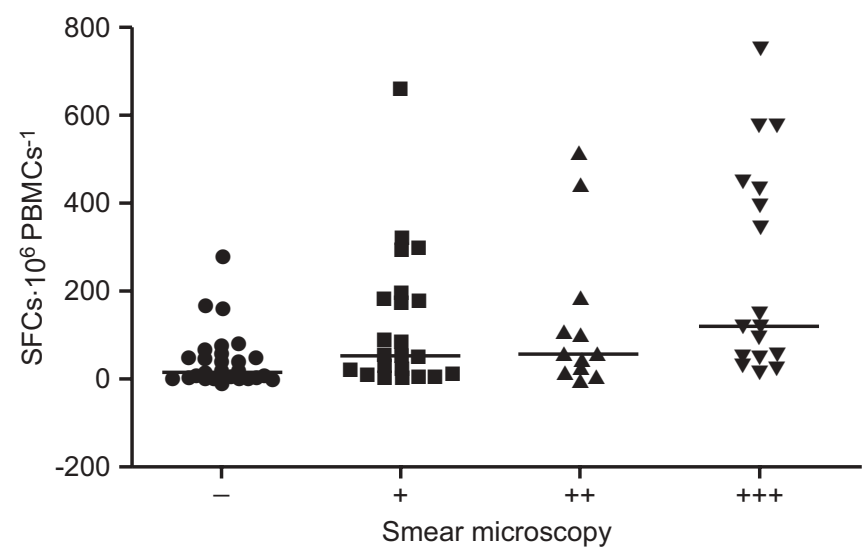

FIGURE 3. Scatter plot showing bacillary load-immune response relationship. Data points represent individuals and horizontal bars medians. The quantitative T-cell response was shown to increase with increasing smear positivity $(p=0.0008$; Kruskal-Wallis test). SFC: spot-forming cell; PBMC: peripheral blood mononuclear cell. -: 0 acid-fast bacillus (AFB) 100 oil immersion fields $(\mathrm{OIFs})^{-1} ;$ +: 10 $99 \mathrm{AFBs} \cdot 100 \mathrm{OIFs}^{-1} ;++$ : $1-10 \mathrm{AFBs} \cdot \mathrm{OIF}^{-1} ;+++:>10 \mathrm{AFBs} \cdot \mathrm{OIF}^{-1}$.

\section{Limitations}

The relatively low specificity of the SFC/CD4 ratio, although higher than the T-SPOT.TB assay alone, means that it would be more suitable as a rule-in test for seeking a diagnosis of active TB than as a rule-out test for excluding TB. A higher proportion of active $\mathrm{TB}$ patients had a history of previous $\mathrm{TB}$, potentially biasing this group towards a higher response rate and ratio compared to the non-TB group. However, as the T-SPOT.TB assay is a short overnight assay, the T-cell response is thought to predominantly constitute effector, and not memory, T-cells. Therefore, a previous history of TB, which would induce memory T-cells, should not significantly influence the present results.

Active TB patients were significantly older, with lower CD4 counts and BMIs, as well as a higher proportion of males, than the non-TB group. On multivariate analysis, age, BMI and a low $\mathrm{CD} 4$ count were significant risk factors for active TB. This

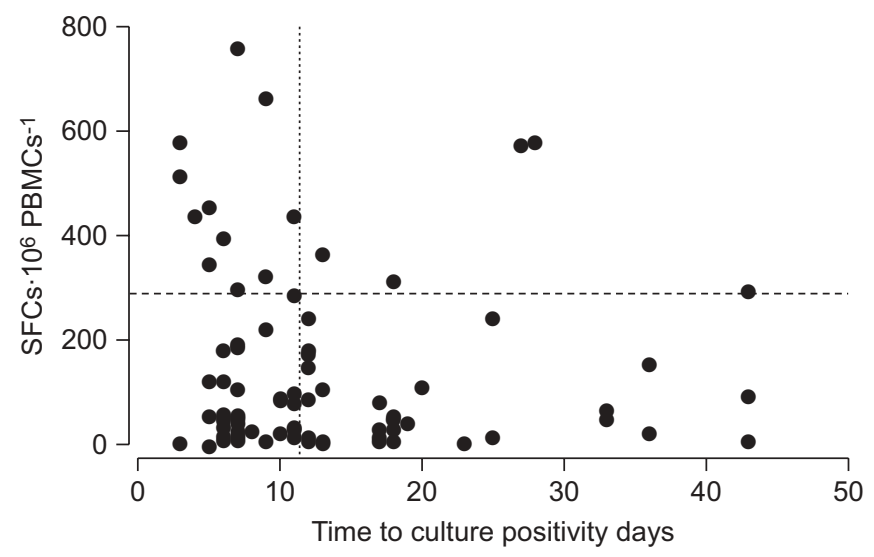

FIGURE 4. Scatter plot showing the number of spot-forming cells (SFCs) and time to culture positivity in active tuberculosis patients (…...... median time to culture positivity; ------: 90th percentile of SFC number) $\left(r_{S}=-0.12 ; p=0.29\right.$ ). PBMC: peripheral blood mononuclear cell. 
difference in baseline characteristics between the two groups could be a potential source of bias.

In relating smear microscopy results to the T-cell response, the present analysis was post hoc, and smear microscopy was performed at reference laboratories as per programmatic guidelines and was not study-specific. As a result, performerrelated variability could affect the validity of the results. Nonetheless, the findings are interesting and warrant further investigation under more controlled conditions.

In addition, frozen cells were used instead of fresh, as recommended by the manufacturer, due to the logistic constraints of the study. As a result, a direct comparison of fresh and frozen cells from 11 patients (frozen for a mean of 2.5 days) was conducted and no difference was shown in the proportion of nonresponders between fresh and frozen samples (data not shown).

In conclusion, in the present setting with a high burden of $\mathrm{TB}$, a PPV of 70\% and NPV of 75\% could limit the ratio's clinical use since $30 \%$ of persons testing positive using the ratio would be unnecessarily treated and $25 \%$ of those testing negative would have a TB diagnosis missed. As a result, the present findings are more easily translated into practice in low TB prevalence areas, where the pre-test probability of LTBI is lower than in the present setting. Nonetheless, the possibility that this novel ratio technique could aid the clinical decision to start $\mathrm{TB}$ treatment, particularly in advanced immunosuppression, and shortening the time to TB treatment, merits further evaluation.

\section{SUPPORT STATEMENT}

This study was supported by grants from the European Union (Brussels, Belgium; EU Sante 121404C/G/Multi) and the National Institutes of Health (Bethesda, MD, USA; NIH 1RO1HD058791-01). R.J. Wilkinson is funded by the Wellcome Trust (London, UK; grants 072070, 084323 and 088316) and Medical Research Council (London, UK). M. Mendelson receives funding support from the President's Emergency Plan for AIDS Relief (Washington, DC, USA)/United States Agency for International Development (Washington, DC, USA).

\section{STATEMENT OF INTEREST}

Statements of interest for J. Patel and R.J. Wilkinson, and for the study itself can be found at www.erj.ersjournals.com $/ \mathrm{misc} /$ statements.dtl

\section{REFERENCES}

1 World Health Organization. Global Tuberculosis Control 2009: Epidemiology. Strategy. Financing: WHO Report 2009. Geneva, World Health Organization, 2009.

2 Wood R, Maartens G, Lombard CJ. Risk factors for developing tuberculosis in HIV-1-infected adults from communities with a low or very high incidence of tuberculosis. J Acquir Immune Defic Syndr 2000; 23: 75-80.

3 Elliott AM, Hodsdon WS, Kyosiimire J, et al. Cytokine responses and progression to active tuberculosis in HIV-1-infected Ugandans: a prospective study. Trans R Soc Trop Med Hyg 2004; 98: 660-670.

4 Sonnenberg P, Glynn JR, Fielding K, et al. How soon after infection with HIV does the risk of tuberculosis start to increase? A retrospective cohort study in South African gold miners. J Infect Dis 2005; 191: 150-158.
5 Elliott AM, Namaambo K, Allen BW, et al. Negative sputum smear results in HIV-positive patients with pulmonary tuberculosis in Lusaka, Zambia. Tuber Lung Dis 1993; 74: 191-194.

6 Huebner RE, Schein MF, Bass JB Jr. The tuberculin skin test. Clin Infect Dis 1993; 17: 968-975.

7 Duncan LE, Elliott AM, Hayes RJ, et al. Tuberculin sensitivity and HIV-1 status of patients attending a sexually transmitted diseases clinic in Lusaka, Zambia: a cross-sectional study. Trans $R$ Soc Trop Med Hyg 1995; 89: 37-40.

8 Clark SA, Martin SL, Pozniak A, et al. Tuberculosis antigenspecific immune responses can be detected using enzyme-linked immunospot technology in human immunodeficiency virus (HIV)-1 patients with advanced disease. Clin Exp Immunol 2007; 150: 238-244.

9 Chapman AL, Munkanta M, Wilkinson KA, et al. Rapid detection of active and latent tuberculosis infection in HIV-positive individuals by enumeration of Mycobacterium tuberculosis-specific T cells. AIDS 2002; 16: 2285-2293.

10 Mandalakas AM, Hesseling AC, Chegou NN, et al. High level of discordant IGRA results in HIV-infected adults and children. Int $J$ Tuberc Lung Dis 2008; 12: 417-423.

11 Rangaka MX, Wilkinson KA, Seldon R, et al. Effect of HIV-1 infection on T-cell-based and skin test detection of tuberculosis infection. Am J Respir Crit Care Med 2007; 175: 514-520.

12 Rangaka MX, Diwakar L, Seldon R, et al. Clinical, immunological, and epidemiological importance of antituberculosis $\mathrm{T}$ cell responses in HIV-infected Africans. Clin Infect Dis 2007; 44: 1639-1646.

13 Oxford Immunotec. T-SPOT๕.TB. www.oxfordimmunotec.com Date last accessed: July 6, 2009.

14 Chen $X$, Yang $Q$, Zhang $M$, et al. Diagnosis of active tuberculosis in China using an in-house gamma interferon enzyme-linked immunospot assay. Clin Vaccine Immunol 2009; 16: 879-884.

15 Chee CB, Gan SH, Khinmar KW, et al. Comparison of sensitivities of two commercial gamma interferon release assays for pulmonary tuberculosis. J Clin Microbiol 2008; 46: 1935-1940.

16 Nishimura $T$, Hasegawa $N$, Mori $M$, et al. Accuracy of an interferon- $\gamma$ release assay to detect active pulmonary and extrapulmonary tuberculosis. Int J Tuberc Lung Dis 2008; 12: 269-274.

17 Kang YA, Lee HW, Hwang SS, et al. Usefulness of whole-blood interferon- $\gamma$ assay and interferon- $\gamma$ enzyme-linked immunospot assay in the diagnosis of active pulmonary tuberculosis. Chest 2007; 132: 959-965.

18 Dewan PK, Grinsdale J, Kawamura LM. Low sensitivity of a whole-blood interferon- $\gamma$ release assay for detection of active tuberculosis. Clin Infect Dis 2007; 44: 69-73.

19 Syed Ahamed Kabeer B, Sikhamani R, Swaminathan S, et al. Role of interferon gamma release assay in active TB diagnosis among HIV infected individuals. PLoS One 2009; 4: e5718.

20 Ewer K, Deeks J, Alvarez L, et al. Comparison of T-cell-based assay with tuberculin skin test for diagnosis of Mycobacterium tuberculosis infection in a school tuberculosis outbreak. Lancet 2003; 361: $1168-1173$.

21 Hill PC, Fox A, Jeffries DJ, et al. Quantitative T cell assay reflects infectious load of Mycobacterium tuberculosis in an endemic case contact model. Clin Infect Dis 2005; 40: 273-278.

22 Ribeiro S, Dooley K, Hackman J, et al. T-SPOT.TB responses during treatment of pulmonary tuberculosis. BMC Infect Dis 2009; 9: 23.

23 Goletti D, Carrara S, Mayanja-Kizza H, et al. Response to $M$. tuberculosis selected RD1 peptides in Ugandan HIV-infected patients with smear positive pulmonary tuberculosis: a pilot study. BMC Infect Dis 2008; 8: 11.

24 Aiken AM, Hill PC, Fox A, et al. Reversion of the ELISPOT test after treatment in Gambian tuberculosis cases. BMC Infect Dis 2006; 6: 66 . 